\title{
Constraining the Randall-Sundrum Model Using Diphoton Production at Hadron Colliders
}

\author{
K. Sridhar \\ Department of Theoretical Physics, \\ Tata Institute of Fundamental Research, \\ Homi Bhabha Road, Bombay 400 005, India.
}

\begin{abstract}
Virtual effects of gravitons in the production of diphotons at the upgraded Tevatron and at the LHC are analysed with the idea of probing the parameter space of the RandallSundrum (RS) model. It is shown that this process yields stringent constraints on the parameter space of the RS model. We show that data on diphoton production from Tevatron Run II will be sensitive to a masses of the first graviton resonance in the range of $700-1150 \mathrm{GeV}$, while at LHC the mass range probed will be in the region of $3.5-5.5 \mathrm{TeV}$.
\end{abstract}

*sridhar@theory.tifr.res.in 
The physics of extra spacetime dimensions and its implications for high energy physics and for astrophysics and cosmology has attracted tremendous attention in recent years. The observable universe is a dynamical hypersurface: a $D_{3}$-brane (or 3 -brane) existing in a higher dimensional spacetime. The Standard Model (SM) fields are localized on the brane but gravity can propagate in the bulk. In such scenarios, the gravity/string scale can be lowered down from the Planck scale to the TeV scale [1]. For high energy physics this is exciting because it provides fresh perspectives to the solution of the hierarchy problem and also suggests the discovery of new physics at $\mathrm{TeV}$-scale colliders.

The first realization of these ideas is the ADD scenario proposed by Arkani-Hamed, Dimopoulos and Dvali [2], where, starting from a higher dimensional theory, an effective four-dimensional theory at a scale $M_{S} \sim \mathrm{TeV}$ is obtained. This is done by compactifying the extra dimensions to magnitudes which are large compared to the Planck length. The compactification radii in the ADD scenario can vary from a fermi to a millimetre, depending upon the number of large dimensions [3]. While the ADD model does not run into any obvious conflict with existing experimental data, it predicts large deviations from the SM in several phenomena which can be observed at existing and future high-energy colliders [4]. Thus, laboratory data can be used to derive bounds on $M_{S}$ in the TeV range. Bounds on this model have also been derived from cosmological and astrophysical considerations and some these are considerably stronger than a TeV [5].

The main problem that one faces within the ADD model is the reappearance of disparate scales viz., the string scale $M_{S} \sim 1 \mathrm{TeV}$ and the compactification radius $R_{c} \sim\left(10^{-16} \mathrm{TeV}\right)^{-1}$. The stability of these lrage dimensions is an undesirable feature of this model and it was in an attempt to resolve this issue that the Randall-Sundrum (RS) model originated [6]. In its original form, the $\mathrm{RS}$ model is a five dimensional model where the fifth dimension $\phi$ is compactified on a $\mathbf{S}^{1} / \mathbf{Z}^{2}$ orbifold with a radius $R_{c}$ which is somewhat larger than the Planck length. At the orbifold fixed points, $\phi=0, \pi$, two 3-branes called the Planck brane and the TeV brane are located. The $\mathrm{SM}$ fields are assumed to be localised on the $\mathrm{TeV}$ brane. To get Poincaré invariance on the brane, it is necessary to fine-tune the cosmological constants both on the brane and in the bulk. The model proposes a novel five-dimensional metric of the form

$$
d s^{2}=e^{-\mathcal{K} R_{c} \phi} \eta_{\mu \nu} d x^{\mu} d x^{\nu}+R_{c}^{2} d \phi^{2}
$$

This metric is non-factorizable or warped and the exponential warp factor $e^{-\mathcal{K} R_{c} \phi}$ serves as a conformal factor for fields localised on the brane and this can be used to solve the hierarchy problem. The huge ratio $\frac{M_{P}}{M_{E W}} \sim 10^{15}$ can be generated by the exponent $\pi \mathcal{K} R_{c}$ which needs to be only of $\mathrm{O}(30) . R_{c}$ can be stabilised against quantum fluctuations either by introducing an extra scalar field in the bulk [7, 8], or by invoking supersymmetry [9].

To derive the consequences of the RS model, a linearised gravity approach is used where the curved metric is approximated by fluctuations $h_{\mu \nu}$ about its Minkowski 
value. On compactification of the extra dimensions, a tower of massive Kaluza-Klein $(\mathrm{KK})$ excitations of the graviton, $h_{\mu \nu}^{(\vec{n})}$, result on the 3 -brane. The interactions of these with the SM particles are given by:

$$
\mathcal{L}_{i n t}=-\frac{1}{\bar{M}_{P}} T^{\mu \nu}(x) h_{\mu \nu}^{(0)}(x)-\frac{e^{\pi \mathcal{K} R_{c}}}{\bar{M}_{P}} \sum_{1}^{\infty} T^{\mu \nu}(x) h_{\mu \nu}^{(n)}(x),
$$

where $\bar{M}_{P}=M_{P} / \sqrt{8 \pi}$ is the reduced Planck mass and $T^{\mu \nu}$ is the symmetric energymomentum tensor for the observable particles on the 3-brane, computed using the flat space metric. The masses of the $h_{\mu \nu}^{(\vec{n})}$ are given by

$$
M_{n}=x_{n} \mathcal{K} e^{-\pi \mathcal{K} R_{c}}
$$

where the $x_{n}$ are the zeros of the Bessel function $J_{1}(x)$ of order unity [8]. The masses of the KK excitations are not evenly spaced in this model. The zero-mode in the tower of excitations essentially decouples because of its weak coupling but the couplings of the massive RS gravitons are enhanced by the exponential $e^{\pi \mathcal{K} R_{c}}$ leading to interactions of electroweak strength. The Feynman rules in this model are essentially the same as those worked out [10, 11] for the ADD case, except for the overall warp factor in the RS case.

The basic parameters of the RS model are

$$
\begin{aligned}
m_{0} & =\mathcal{K} e^{-\pi \mathcal{K} R_{c}} \\
c_{0} & =\mathcal{K} / M_{P}
\end{aligned}
$$

where $m_{0}$ is a scale of the dimension of mass and sets the scale for the masses of the KK excitations, and $c_{0}$ is an effective coupling. The interaction of massive KK gravitons with matter can be written as

$$
\mathcal{L}_{\text {int }}=-\sqrt{8 \pi} \frac{c_{0}}{m_{0}} \sum_{n}^{\infty} T^{\mu \nu}(x) h_{\mu \nu}^{(n)}(x) .
$$

It is expected that the parameter $c_{0}$ lies in the range $[0.01,0.1]$. This is because the scale $\mathcal{K}$ is related to the curvature of the fifth dimension and so the upper bound on $c_{0}$ results if we want to avoid strong curvature effects. But at the same time we would not want $\mathcal{K}$ to be too small as compared to $\bar{M}_{P}$, since that would introduce a new hierarchy. Values of $m_{0}$ are determined in terms of $\mathcal{K} R_{c} \sim 10$, so that $m_{0}$ ranging from about a $100 \mathrm{GeV}$ to a $\mathrm{TeV}$ are possible. We would like to emphasise here that $m_{0}$ cannot become arbitrarily large in the $\mathrm{RS}$ model. This is because if $m_{0}$ becomes very large, it would require either $\mathcal{K}$ to be large, or $\mathcal{K} R_{c}$ to be small (see Eq. (1). This results in a large curvature of the fifth dimension which makes it difficult to fine-tune the cosmological constants on the brane and the bulk to get a flat metric on the $\mathrm{TeV}$ brane. Consequently, the natural mass for the first graviton excitation is at most of the order of a few TeV. 
It is interesting to ask what is the kind of collider phenomenology that results with the RS model. Because of the fact that the zero mode decouples, it is only the heavier modes one can hope to detect in experiments. In the fortuitous circumstance that these modes are within the reach of high-energy experiments, interesting effects like resonance production can be observed, with the resonance decaying within the detectors. If this is not the case and if the the gravitons are heavier then the best strategy will be to look for the virtual effects of the gravitons on observables measured in high-energy collider experiments. Indeed, some of the phenomenology of resonant production of the KK excitations and the virtual effects have already been studied in processes like dilepton production at hadron colliders [12], $t \bar{t}$ production at hadron colliders [13 and in deep-inelastic scattering at HERA [14]. Novel effects like probing strong gravity via black-hole production at low energies have also been discussed in the context of the RS model 15.

In this letter, we study the virtual effects of the exchange of spin-2 KK modes, in the RS model, in diphoton production at the Tevatron and the LHC. In experiments, these photons are identified as isolated energy deposits in the electromagnetic calorimeter. Selection cuts are then applied to improve the signal-to-noise ratio. The D0 and CDF collaborations have studied diphoton production in Run I of the Tevatron experiment. The D0 collaboration has used these diphoton production data to derive constraints on the ADD model [16]. The Run I studies are however limited by statistics and for the purposes of the present paper of limited interest. In this paper, therefore, we concentrate on the production of diphotons at Run II of the Tevatron and at the LHC. Because of the high statistics that will be achieved in these experiments, it will be possible to perform a detailed study of mass and angular distributions and derive possibly stringent constraints on the parameter space of the RS model.

The cross-sections for the $q \bar{q} \rightarrow \gamma \gamma$ and $g g \rightarrow \gamma \gamma$ subprocesses are [17, 18]:

$$
\begin{aligned}
\frac{d \hat{\sigma}}{d \hat{t}}(q \bar{q} \rightarrow \gamma \gamma)= & \frac{2 \pi \alpha^{2} Q_{q}^{4}}{3 \hat{s}^{2}} \frac{1+\cos ^{2} \theta^{*}}{1-\cos ^{2} \theta^{*}} \\
& +\frac{\alpha Q_{q}^{2}}{96 \pi} \operatorname{Re}\left[C\left(x_{s}\right)\right]\left(1+\cos ^{2} \theta^{*}\right)+\frac{s^{2}}{24576 \pi}\left|C\left(x_{s}\right)\right|^{2}\left(1-\cos ^{4} \theta^{*}\right),
\end{aligned}
$$

and

$$
\frac{d \hat{\sigma}}{d \hat{t}}(g g \rightarrow \gamma \gamma)=+\frac{s^{2}}{65536 \pi}\left|C\left(x_{s}\right)\right|^{2}\left(1+6 \cos ^{2} \theta^{*}+\cos ^{4} \theta^{*}\right) .
$$

The SM box contribution $g g \rightarrow \gamma \gamma$ makes a very small contribution at the Tevatron energy and is negligible [18]. At the LHC energy, this box contribution is somewhat increased because of the initial gluon flux but, as shown in Ref. [18], in spite of this increase this contribution is an order of magnitude smaller than the SM $q \bar{q} \rightarrow \gamma \gamma$ contribution for diphoton invariant mass of $500 \mathrm{GeV}$ and is more than two orders of magnitude smaller for diphoton invariant mass greater than about $1750 \mathrm{GeV}$. On the 
other hand, the new physics effects dominate in the large invariant mass bins and, therefore, in the invariant mass region of interest the SM box contribution is negligible even for the case of the LHC.

In the above equations, $\cos \theta^{*}$ is the scattering angle in the partonic c.m. frame, $x_{s} \equiv \frac{\sqrt{\hat{s}}}{m_{0}}$ and $C(x)$ is defined as

$$
C_{(x)}=\frac{32 \pi c_{0}^{2}}{m_{0}^{4}} \lambda(x)
$$

with

$$
\lambda\left(x_{s}\right)=m_{0}^{2} \sum_{n} \frac{1}{\hat{s}-M_{n}^{2}+i M_{n} \Gamma_{n}} .
$$

and the $M_{n}$ are the masses of the individual resonances and the $\Gamma_{n}$ are the corresponding widths. The graviton widths are obtained by calculating their decays into final states involving SM particles. This gives

$$
\Gamma_{n}=m_{0} c_{0}^{2} x_{n}^{3} \Delta_{n}
$$

where

$$
\Delta_{n}=\Delta_{n}^{\gamma \gamma}+\Delta_{n}^{g g}+\Delta_{n}^{W W}+\Delta_{n}^{Z Z}+\sum_{\nu} \Delta_{n}^{\nu \nu}+\sum_{l} \Delta_{n}^{l l}+\sum_{q} \Delta_{n}^{q q}+\Delta_{n}^{H H}
$$

and each $\Delta_{n}^{a a}$ is a numerical coefficient arising in the decay $h^{n} \rightarrow a \bar{a}$. For the partial width $\Delta_{n}^{H H}$, we have fixed $M_{H}=250 \mathrm{GeV}$ in our numerical studies.

Given the masses and the widths of the individual graviton resonances, we have to sum over all the resonances to get the value of $\lambda\left(x_{s}\right)$. We perform this sum numerically, using the fact that the higher zeros of the Bessel function become evenly-spaced. For a given value of $x_{s}=\frac{\sqrt{s}}{m_{0}}$, we retain all resonances which contribute with a significance greater than one per mil, and treat the remaining KK modes as virtual particles (in which case the sum can be done analytically).

Using the above sub-process cross-sections we can compute the diphoton invariant mass distribution, $d \sigma / d M$ and the double differential cross-section $d \sigma / d M d \cos \theta^{*}$ for $\gamma \gamma$ production cross-section at the Tevatron and the LHC, by convoluting with parton densities. For our numerical studies, we have used the CTEQ4M parametrisations [19] for the parton distributions. To obtain the bounds on the $m_{0}-c_{0}$ parameter space, we compute the cross-section for the diphoton production process in different bins of $M$ (or $M$ and $\cos \theta^{*}$, for the case of the double differential cross-section. Assuming Poisson-distributed data, we then compute the $\chi^{2}$ using the following expression:

$$
\chi^{2}\left(m_{0}, c_{0}\right)=\sum_{i=\text { bins }}\left[2\left(n_{i}^{\text {th }}-n_{i}^{\text {obs }}\right)+2 n_{i}^{\text {obs }} \ln \left(\frac{n_{i}^{\text {obs }}}{n_{i}^{\text {th }}}\right)\right],
$$


where $n_{i}^{\text {obs }}$ is taken to be the SM prediction for the number of events in the bin $i$ and $n_{i}^{\text {th }}$ is the prediction obtained by adding the new physics contribution to the SM expectation. The $\chi^{2}$ so calculated is used to obtain a $95 \%$ C.L. constraint on the $m_{0}-c_{0}$ parameter space. For Tevatron Run II, we use the following parameters: $\sqrt{s}=2 \mathrm{TeV}$, $\mathcal{L}=2 \mathrm{fb}^{-1}$, bin size in $M=80 \mathrm{GeV}$ for $50<M<610 \mathrm{GeV}$ and $M=610--1500 \mathrm{GeV}$ is combined into one bin, $y^{\gamma}<|1.2|$ for each of the photons. When computing the double differential cross-section $d \sigma / d M / d \cos \theta^{*}$, we use a bin size of 0.2 for $-1<\cos \theta^{*}<1$. For the LHC we use the following parameters: $\sqrt{s}=14 T e V, \mathcal{L}=100 \mathrm{fb}^{-1}$, bin size in $M=200 \mathrm{GeV}$ for $500<M<3250 \mathrm{GeV}$ and $M=3250--5000 \mathrm{GeV}$ is combined into one bin, $y^{\gamma}<|2.5|$ for each of the photons. NLO QCD corrections to the SM contribution to the diphoton cross-section yields a K-factor of 1.3 (1.1) for Tevatron (LHC) [20]; we assume same K-factor for the new physics contribution and simply multiply the full cross-section by the K-factor to account for the effect of higher-order QCD corrections.

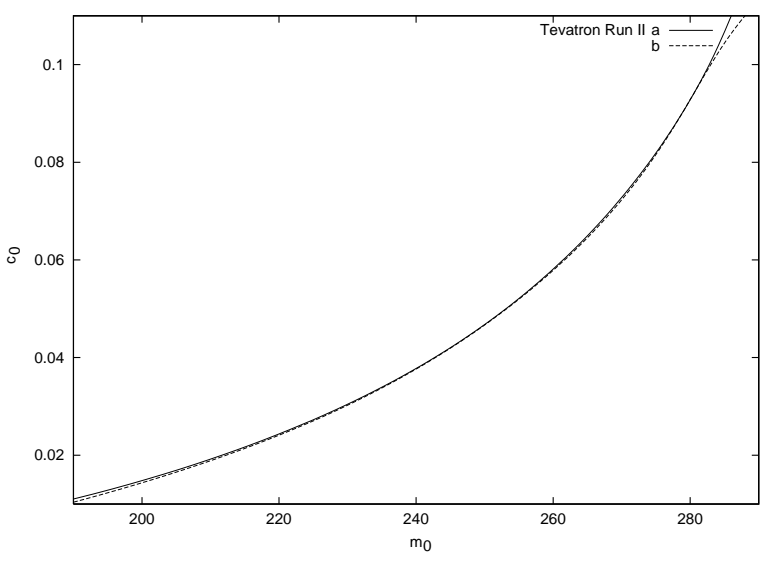

Figure 1: Constraints on the $m_{0}-c_{0}$ plane of the Randall-Sundrum model, using $\gamma \gamma$ production at Run II of the Tevatron.

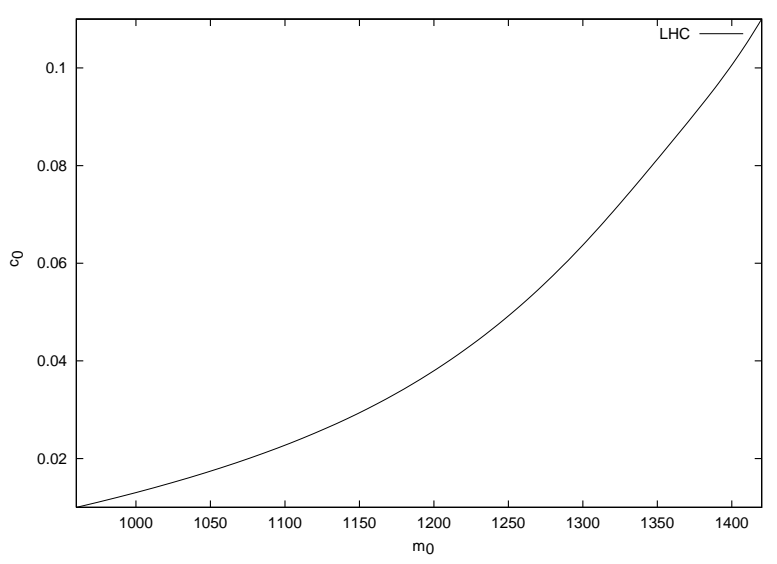

Figure 2: Constraints on the $m_{0}-c_{0}$ plane of the Randall-Sundrum model, using $\gamma \gamma$ production at the $\mathrm{LHC}$.

We present the results of our computations in Fig. 1. In Fig 1(a), we show the excluded region at $95 \%$ C.L. in the $\left(m_{0}, c_{0}\right)$ plane obtained by computing the $\chi^{2}$ as described above. The two curves shown in the figure are obtained by considering the $d \sigma / d M$ and the $d \sigma / d M d \cos \theta^{*}$ distributions. We find that the invariant mass distribution already gives strong constraints but the inclusion of the information on angular distribution does not improve these constraints. We find that for values of $c_{0}$ between 0.01 and 0.1 , the $95 \%$ C.L. limits on $m_{0}$ that can be derived at the Run II of the 
Tevatron vary between $180 \mathrm{GeV}$ and $290 \mathrm{GeV}$. This means that the first KK graviton resonance must lie above $700 \mathrm{GeV}$ at the least. For the LHC, the constraints are shown in Fig. 1(b). We find that the accessible $95 \%$ C.L. limits on $m_{0}$ for $0.01<c_{0}<0.1$ lie in the range between $950 \mathrm{GeV}$ and $1450 \mathrm{GeV}$, which means that the first graviton resonance can be probed up to around $3.5 \mathrm{TeV}$. As discussed earlier, the mass of the first graviton excitation is at most of the order a few $\mathrm{TeV}$, and it is an exciting prospect that this is the range of graviton masses is likely to be probed by the diphoton production process at the LHC.

To summarize, we have analysed the effects of the interactions of the spin-2 KaluzaKlein modes with SM fields in diphoton production at the upgraded Tevatron (Run II) and at the LHC, in the context of the Randall-Sundrum model. By analysing the invariant mass distributions of the photon pair, we derive $95 \%$ C.L. limits on $m_{0}$ for $0.01<c_{0}<0.1$. These limits are between 180 and $290 \mathrm{GeV}$ for the Tevatron Run II and between 950 and $1450 \mathrm{GeV}$ for the LHC. The range of $m_{0}$ values that will be probed by this process at the LHC are such that there is the exciting possibility of detecting the virtual effects at the LHC or, conversely, non-observation of these graviton modes at the LHC would seriously constrain the Randall-Sundrum model, at least in its simplest form.

\section{References}

[1] P. Horava and E. Witten, Nucl. Phys. B460 (1996) 506; J.D. Lykken Phys. Rev. D54 (1996) 3697; E. Witten, Nucl. Phys. B471 (1996) 135.

[2] N. Arkani-Hamed, S. Dimopoulos and G. Dvali, Phys. Lett. B249 (1998) 263; I. Antoniadis, N. Arkani-Hamed, S. Dimopoulos and G. Dvali, Phys. Lett. B436 (1998) 257.

[3] For a review of the ADD model, see I. Antoniadis and K. Benakli, Int. J. Mod. Phys A15 (2000) 4237; A. Perez-Lorenzana, Univ. of Maryland Preprint No. UMD-PP-00-088 (2000) hep-ph/0008333.

[4] For a review of the phenomenology of the ADD model, see K. Sridhar, Int. J. Mod. Phys A15 (2000) 2397 (hep-ph/0004053); S. Raychaudhuri, Talk given at the Sixth Workshop on High-Energy Phenomenology, Chennai (India), January 2000. For details of the phenomenology see Ref. [21].

[5] S. Cullen and M. Perelstein, Phys. Rev. Lett. 83 (1999) 268; V. Barger et al., Phys. Lett. B461 (1999) 34.

[6] L. Randall and R. Sundrum, Phys. Rev. Lett. 83 (1999) 3370. 
[7] C. Csaki, M. Graesser, L. Randall and J. Terning, Phys. Rev. D 62 (1999) 045015, C. Csaki, M. Graesser and G.D. Kribbs, Santa Cruz Preprint No. SCIPP-00-27, hep-th/0008151 (2000).

[8] W.D. Goldberger and M.B. Wise, Phys. Rev. Lett. 83 (1999) 4922; Phys.Lett. B475 (2000) 275.

[9] R. Altendorfer, J. Bagger and D. Nemeschansky, CITUSC-00-015, hepth/0003117.

[10] G. F. Giudice, R. Rattazzi and J. D. Wells, Nucl. Phys. B544 (1999) 3.

[11] T. Han, J. D. Lykken and R-J. Zhang, Phys. Rev. D59 (1999) 105006.

[12] H. Davoudiasl, J.L. Hewett and T.G. Rizzo, Phys. Rev. Lett 84 (2000) 2080; SLAC Preprint SLAC-PUB-8436 (2000) hep-ph/0006041.

[13] S. Lola, Prakash Mathews, Sreerup Raychaudhuri and K. Sridhar, CERN Preprint No. CERN-TH-2000-275 (2000) hep-ph/0010010.

[14] P. Das, S. Raychaudhuri and S. Sarkar, JHEP 0007:050 (2000).

[15] S.B. Giddings and E. Katz, MIT Preprint No. MIT-CTP-3024 (2000) hepth/0009176.

[16] K. Cheung and G. Landsberg, Phys. Rev. D62 (2000) 076003; B. Abbott et al., Phys. Rev. Lett. 86 (2001) 1156.

[17] K. Cheung, Phys. Rev. D61 (2000) 015005.

[18] O.J.P. Éboli et al., Phys. Rev. D61 (2000) 094007.

[19] H.L. Lai et al., Phys. Rev. D51 (1995) 4763.

[20] B. Bailey, J.F. Owens and J. Ohnemus, Phys. Rev. D46 (1992) 2018.

[21] Prakash Mathews, Sreerup Raychaudhuri and K. Sridhar, Phys. Lett. B 450 (1999) 343; Phys. Lett. B 455 (1999) 115; JHEP 0007:008 (2000). 